\title{
Interaction of higher-dimensional rotating black holes with branes
}

\author{
Valeri P Frolov ${ }^{1}$, Dmitri V Fursaev ${ }^{2}$ and Dejan Stojković ${ }^{3}$ \\ 1 Theoretical Physics Institute, Department of Physics, University of Alberta, \\ Edmonton T6G 2J1, Canada \\ 2 Joint Institute for Nuclear Research, Bogoliubov Laboratory of Theoretical Physics, \\ 141980 Dubna, Russia \\ ${ }^{3}$ MCTP, Department of Physics, University of Michigan, Ann Arbor, MI, USA \\ E-mail: frolov@phys.ualberta.ca,fursaev@thsun1.jinr.ru and dejans@umich.edu
}

Received 13 March 2004

Published 23 June 2004

Online at stacks.iop.org/CQG/21/3483

doi:10.1088/0264-9381/21/14/011

\begin{abstract}
We study interaction of rotating higher-dimensional black holes with a brane in spacetimes with large extra dimensions. We demonstrate that in a general case a rotating black hole attached to a brane can lose bulk components of its angular momenta. A stationary black hole can have only those components of the angular momenta which are connected with Killing vectors generating transformations preserving a position of the brane. In a final stationary state the null Killing vector generating the black hole horizon is tangent to the brane. We discuss first the interaction of a cosmic string and a domain wall with the 4D Kerr black hole. We then prove the general result for slowly rotating higher-dimensional black holes interacting with branes. The characteristic time when a rotating black hole with gravitational radius $r_{0}$ reaches this final stationary state is $T \sim r_{0}^{p-1} /(G \sigma)$, where $G$ is the higher-dimensional gravitational coupling constant, $\sigma$ is the brane tension and $p$ is the number of extra dimensions.
\end{abstract}

PACS numbers: 04.70.Bw, 97.60.Lf, 11.25.-w

\section{Introduction}

Models [1, 2] where a spacetime has large or infinite extra dimensions became very popular recently. In these models, the fundamental energy scale can be as low as a few $\mathrm{TeV}$ which opens the possibility for experimental tests of predictions of these models in future collider and cosmic ray experiments $[3,4]$. In a higher-dimensional space (with a number of extra dimensions $p$ ) at distances $r$ much smaller than the size of extra dimensions, the 
gravitational force $G M / r^{1+p}$ becomes much stronger than in 4D space. For this reason in the interaction of particles with $\mathrm{TeV}$ energies, one can expect considerable emission of bulk gravitons. This effect could be observed as the energy-momentum non-conservation in the braneworld. Another dramatic prediction of these models is a possibility of creation of mini black holes when the centre of mass energy of two colliding particles becomes higher than fundamental energy [3].

Being gravitational solitons, black holes can propagate in the bulk space and thus serve as probes of extra dimensions. We focus our attention on black holes with a size much smaller than the size of the extra dimensions. In this case the effects of the extra dimensions on the black hole geometry are small and can be neglected. (For a recent review on black holes in a spacetime with large extra dimensions, see [5].)

In a general case, a black hole created by the collision of two particles is rotating. If there is no emission of the bulk gravitons the black hole rotation occurs within the brane. In a more realistic situation when bulk gravitons are emitted, a black hole can also acquire bulk components of the angular momentum. There are also other processes which may result in black hole rotation in the bulk dimensions, for example, if a black hole collides with a particle or another black hole with emission of bulk gravitons, or when it emits bulk gravitons in the Hawking evaporation process.

In the approximation when the tension of the brane is neglected and a black hole gravitational radius is much smaller than the size of the extra dimensions, the higherdimensional rotating black holes are described by the Myers-Perry (MP) metric [6]. ${ }^{4}$ In this paper we discuss the effects of interaction of a rotating higher-dimensional black hole with a brane. We shall make two simplifying assumptions: (i) the brane-black hole interaction is weak; (ii) the black hole is slowly rotating. The first assumption means that we take into account corrections linear in terms of the brane tension. The second assumption implies that we shall study only linear in the rotation parameter corrections. In this approach, we can start with a static unperturbed solution for a test brane in a spacetime of a non-rotating black hole (section 2) and consider the perturbation of the brane induced by a small rotation of the black hole. There exists an evident static solution of Nambu-Goto equations describing a static thin brane, namely a configuration when the worldsheet representing the brane coincides with the 'equatorial plane' of the black hole. The equatorial plane of a spherically symmetric spacetime is invariant under a reflection transforming the northern hemisphere into the southern one and thus it is a geodesic sub-manifold. Since any geodesic surface is also a minimal one, this equatorial plane obeys the Nambu-Goto equations.

We start our study of interaction of slowly rotating black holes with branes by considering the simplest example in 4D space, namely, a thin domain wall interacting with the Kerr black hole (section 3). According to the well-known result proved by Hawking in 1972 [9] a stationary black hole either does not rotate and its metric is static, or it rotates and the spacetime possesses additional axial symmetry. In the latter case, the matter distribution around a stationary rotating black hole must be axially symmetric, which means that the Lie derivative of the matter stress-energy tensor $T^{\mu \nu}$ along the Killing vector field $\xi_{\varphi}$ generating rotations of the black hole is vanishing, $\mathcal{L}_{\xi_{\varphi}} T^{\mu \nu}=0$. This result implies that if there exists a matter distribution violating axial symmetry its gravitational interaction with the black hole slows down its rotation until it becomes static.

If a black hole has a rotation around the axis orthogonal to the domain wall, the configuration is axisymmetric and that there is no angular momentum transfer from the black

\footnotetext{
4 It should be emphasized that in the higher-dimensional case a black hole's horizon can have the topology which differs from the topology of the sphere [7] and rapidly rotating black holes can be unstable [8]. For simplicity we do not consider these effects.
} 
hole to the domain wall in this case. The situation is quite different when the axis of rotation is not orthogonal to the unperturbed domain wall. A system loses its axial symmetry, and one can expect the angular momentum transfer from a black hole to the brane. In section 3 we calculate the rate of the angular momentum flux.

It is interesting that in the case of a cosmic string, a solution for a stationary test string attached to the black hole is known exactly for an arbitrary value of the rotation parameter. Using this solution, it is possible to demonstrate that a rotating black hole attached to the string loses its angular momentum. We calculate the rate of the angular momentum loss. This process continues until the system reaches a final stationary state where the string coincides with the axis of rotation of the black hole. These results are presented in the appendix.

In the second part of the paper, we discuss the interaction of rotating black holes with branes in a spacetime with large extra dimensions. In many aspects this generalization is straightforward. One only should remember that in a higher-dimensional case there are several mutually commutative and orthogonal spacelike Killing vectors $\xi_{(i)}$ singled out by the property that they have closed integral lines. The rotation of the black hole is characterized by a set of angular velocities $\Omega_{i}$ of the horizon along corresponding integral lines of $\xi_{(i)}$. We demonstrate that a slowly rotating higher-dimensional black hole interacting with a static brane loses some of the components of its angular momenta and reaches a final stationary equilibrium state. In this state the stress-energy tensor of the brane satisfies the following condition $\sum_{i} \Omega_{i} \mathcal{L}_{\xi_{(i)}} T^{\mu \nu}=0$. The final state also possesses the property that the null Killing vector generating the black hole horizon is tangent to the brane, which means that there is no energy fluxes through the black hole horizon.

Our calculations show that the characteristic time of relaxation during which a rotating black hole reaches the equilibrium state is shorter than the time during which it loses its bulk angular momentum because of Hawking radiation. This may be an important experimental signature of mini black holes in future collider and cosmic ray experiments.

We conclude the paper by considering special cases of mutual orientation of a rotating black hole and brane, and illustrate the general conclusions (section 6). In the discussion (section 7) we comment on brane-black hole interaction in Randall-Sundrum braneworlds.

\section{A black hole attached to a brane}

Before discussing the effects connected with the rotation of black holes, we consider a simpler case of a static brane attached to a non-rotating black hole. In the approximation when the gravitational back-reaction of the brane is neglected its worldsheet obeys the Nambu-Goto equation

$$
{ }^{(n+1)} \Delta X^{\mu}+\gamma^{a b} \Gamma^{\mu}{ }_{\nu \lambda} X_{, a}^{\nu} X_{, b}^{\lambda}=0 .
$$

The relations $X^{\mu}=X^{\mu}\left(\zeta^{a}\right)$ determine the embedding of the $(n+1)$-dimensional brane into $(N+1)$-dimensional bulk spacetime. $\zeta^{a},(a, b=0, n)$ are internal coordinates in the brane, and $X^{\mu},(\mu=0, N)$ are coordinates in the bulk space with the metric $g_{\mu \nu}$. The connections $\Gamma_{\nu \lambda}^{\mu}$ are determined for $g_{\mu \nu}$. To exclude degenerate cases, we assume that $0<n<N .{ }^{(n+1)} \Delta$ is the box operator for the induced metric

$$
\gamma_{a b}=g_{\mu \nu} X_{, a}^{\mu} X_{, b}^{\nu} .
$$

The stress-energy tensor of the brane is defined as follows:

$$
\sqrt{-g} T^{\mu \nu}=-\sigma \int \mathrm{d}^{n+1} \zeta \delta^{(N+1)}(X-X(\zeta)) \sqrt{-\gamma} \gamma^{a b} X_{, a}^{\mu} X_{, b}^{v},
$$

where $\sigma$ is the tension of the brane. 
The metric Tangherlini of a non-rotating higher-dimensional black hole is [10]

$$
\mathrm{d} \bar{s}^{2}=-B \mathrm{~d} v^{2}+2 \mathrm{~d} r \mathrm{~d} v+r^{2} \mathrm{~d} \Omega_{N-1}^{2}, \quad B=1-\left(\frac{r_{0}}{r}\right)^{N-2} .
$$

The coordinate $v$ is the advanced time and $\mathrm{d} \Omega_{N-1}^{2}$ is the metric on the unit sphere $S^{N-1}$. For $N=3$ this metric reduces to the Schwarzschild metric. The gravitational radius $r_{0}$ is related to the black hole mass $M$ as follows

$$
M=\frac{(N-1) \mathcal{A}_{N-1}}{16 \pi G_{N+1}} r_{0}^{N-2},
$$

where

$$
\mathcal{A}_{N-1}=\frac{2 \pi^{N / 2}}{\Gamma(N / 2)}
$$

is the area of a unit sphere $S^{N-1}$ and $G_{N+1}$ is the $(N+1)$-dimensional gravitational coupling constant which has dimensionality [mass $] /[\text { length }]^{(N-2)}$.

Consider a unit sphere $S^{N-1}$ embedded in a $N$-dimensional Euclidean space $R^{N}$, and let $X^{A},(A=1, \ldots, N)$ be the Cartesian coordinates in $R^{N}$. One can choose these coordinates so that the equations $X^{n+1}=\cdots=X^{N}=0$ determine the $n$-dimensional hyper-surface (brane). This hyper-surface intersects the unit sphere $S^{N-1}$ along a surface $\mathcal{S}$ which has a geometry of a round unit sphere $S^{n-1}$. The surface $\mathcal{S}$ is a higher-dimensional analogue of a 'large circle' on a two-dimensional sphere. In particular, being considered as a sub-manifold of $S^{N-1}$ it has vanishing extrinsic curvatures, and hence is a geodesic sub-manifold. We denote by $\omega^{\alpha}$ coordinates on $\mathcal{S}$, and by $\mathrm{d} \omega_{n-1}^{2}$ the metric on it.

One can construct a solution for a static $n$-brane as follows. We use $\zeta^{a}=\left(v, r, \omega^{\alpha}\right)$ as coordinates on the brane. Then

$$
\mathrm{d} \bar{\gamma}^{2}=-B \mathrm{~d} v^{2}+2 \mathrm{~d} r \mathrm{~d} v+r^{2} \mathrm{~d} \omega_{n-1}^{2}
$$

is the induced geometry on the brane. It is easy to check that such a surface is geodesic and hence is a solution of the Nambu-Goto equations (2.1).

Denote by $\xi^{\mu}$ a Killing vector field and let $J^{b}$ be a conserved quantity corresponding to $\xi^{\mu}$. For a timelike Killing vector $J^{b}=-E^{b}$, where $E^{b}$ is the energy, while for a generator of rotations, $J^{b}$ is the angular momentum of the brane. The flux per unit time $v$ of $J^{b}$ through the surface $r=$ const of the brane is

$$
\dot{J}^{b} \equiv \frac{\mathrm{d} J^{b}}{\mathrm{~d} v}=\int_{r=\text { const }} \sqrt{-g} T^{r v} \xi_{v} \mathrm{~d}^{N-1} \Omega .
$$

Due to the conservation law $T_{; v}^{\mu \nu}=0$ the flux $j^{b}$ does not depend on $r$. Let us denote by $\dot{J}$ the rate of the loss of the energy or angular momentum of the black hole in this process, $\dot{J}=-\dot{J}^{b}$. By using (2.3), (2.8) one finds

$$
\dot{J}=\sigma \int_{r=\text { const }} \mathrm{d}^{n-1} \omega \sqrt{-\gamma} \gamma^{a b} X_{, a}^{\mu} n_{\mu} X_{, b}^{\nu} \xi_{\nu},
$$

where $n_{\mu}=r_{, \mu}$. The integral is taken over an $(n-1)$-dimensional sphere and $\mathrm{d}^{n-1} \omega$ is a measure on a unit sphere $S^{n-1}$.

For a static black hole, since both of the Killing vectors $\partial_{t}$ and $\partial_{\phi}$ are tangent to the surface $r=$ const, the energy and angular momentum of the black hole are constant.

If a black hole is slowly rotating the brane changes its position from $\bar{X}^{\mu}\left(\zeta^{a}\right)$ to $X^{\mu}\left(\zeta^{a}\right)=\bar{X}^{\mu}\left(\zeta^{a}\right)+\delta X^{\mu}\left(\zeta^{a}\right)$. The brane perturbation can be found as a solution of the 
linearized Nambu-Goto equations (2.1)

${ }^{(n+1)} \bar{\Delta} \delta X^{\mu}+\delta\left({ }^{(n+1)} \Delta\right) \bar{X}^{\mu}+2 \bar{\gamma}^{a b} \bar{\Gamma}_{\nu \lambda}^{\mu} \bar{X}_{, a}^{\lambda} \delta X_{, b}^{\nu}+\delta \gamma^{a b} \bar{\Gamma}_{\nu \lambda}^{\mu} \bar{X}_{, a}^{\lambda} \bar{X}_{, b}^{v}+\bar{\gamma}^{a b} \delta \Gamma_{\nu \lambda}^{\mu} \bar{X}_{, a}^{\lambda} \bar{X}_{, b}^{v}=0$.

Here all quantities with a bar correspond to the static black hole. Variations of the connections $\delta \Gamma_{\nu \lambda}^{\mu}$ are due to the difference $\delta g_{\mu \nu}$ between the metric of a static and rotating black hole, $\delta g_{\mu \nu}=g_{\mu \nu}-\bar{g}_{\mu \nu}$. The variations $\delta \gamma_{a b}$ of the metric induced on the brane worldsheet are caused by variations $\delta g_{\mu \nu}$ of the bulk metric and changes in the brane position

$$
\delta \gamma_{a b}=\delta g_{\mu \nu} \bar{X}_{, a}^{\mu} \bar{X}_{, b}^{\nu}+\left(\delta X_{, a}, \bar{X}_{, b}\right)+\left(\bar{X}_{, a}, \delta X_{, b}\right),
$$

where we denote $(A, B)=\bar{g}_{\mu \nu} A^{\mu} B^{\nu}$, the inner product with respect to the static metric. Variation $\delta\left({ }^{(n+1)} \Delta\right)$ in $(2.10)$ is the variation of the box operator under the change of brane metric.

In what follows we shall use the same coordinates $\zeta^{a}=\left(v, r, \omega^{\alpha}\right)$ on the perturbed brane so that the coordinate volume $\mathrm{d}^{n-1} \omega$ in (2.9) remains the same. To obtain the flux for a perturbed case one needs to calculate only variations $\delta X^{\mu}$ and $\delta \gamma_{a b}$ induced by the perturbations.

\section{A Kerr black hole interacting with a thin domain wall}

\subsection{A solution of the perturbed equation}

We first consider a slowly rotating Kerr back hole interacting with a thin domain wall. For a slowly rotating black hole the rotation parameter $a$ is much smaller that the black hole mass $M$. In this limit the Kerr metric (A.1) takes the form

$$
\mathrm{d} s^{2}=\mathrm{d} \bar{s}^{2}-2 a \sin ^{2} \theta \mathrm{d} \varphi\left(\frac{r_{0}}{r} \mathrm{~d} v+\mathrm{d} r\right) .
$$

Here $\mathrm{d} \bar{s}^{2}$ is the Schwarzschild metric

$$
\mathrm{d} \bar{s}^{2}=-B \mathrm{~d} v^{2}+2 \mathrm{~d} r \mathrm{~d} v+r^{2}\left(\sin ^{2} \theta \mathrm{d} \varphi^{2}+\mathrm{d} \theta^{2}\right),
$$

and $B=1-r_{0} / r, r_{0}=2 M$.

Denote by $\alpha$ an angle between the axis of rotation and the brane, then the equation of the unperturbed domain wall is $\varphi=\bar{\varphi}(\theta)$, where

$$
\sin \bar{\varphi}=\tan \alpha \cot \theta
$$

and $\alpha \leqslant \theta \leqslant \pi-\alpha$ for $0 \leqslant \alpha \leqslant \pi / 2$. The induced metric on the worldsheet of such a tilted domain wall is

$$
\mathrm{d} \bar{\gamma}^{2}=-B \mathrm{~d} v^{2}+2 \mathrm{~d} r \mathrm{~d} v+\frac{r^{2} \sin ^{2} \theta}{\sin ^{2} \theta-\sin ^{2} \alpha} \mathrm{d} \theta^{2}
$$

One can check in this case the Nambu-Goto equations (2.1) reduce to one equation

$$
\bar{\varphi}_{, \theta \theta}+2 \cot \theta \bar{\varphi}_{, \theta}+\sin \theta \cos \theta \bar{\varphi}_{, \theta}^{3}=0,
$$

and (3.3) is its solution.

If a black hole is slowly rotating the position of the domain wall becomes $X^{\mu}=\bar{X}^{\mu}+\delta X^{\mu}$. Those variations $\delta X^{\mu}$ which are tangent to the brane can be gauged away. We use the same coordinates $v, r$ and $\theta$ as in the unperturbed case to parametrize the domain wall

$$
v=\hat{v}, \quad r=\hat{r}, \quad \theta=\hat{\theta},
$$

so that the only nontrivial equation specifying a position of the perturbed domain wall is

$$
\varphi=\bar{\varphi}(\hat{\theta})+a \psi(\hat{r}, \hat{\theta}) .
$$


One can check that for this choice of gauge, the corresponding $v, r$ and $\theta$ components of the Nambu-Goto equations (2.10) are satisfied identically. The equation for $\psi$ can be found from the $\varphi$ component of (2.10)

${ }^{(3)} \bar{\Delta} \psi+2 \bar{\gamma}^{a b} \bar{\Gamma}_{\nu \varphi}^{\varphi} \bar{X}_{, a}^{\nu} \psi_{, b}+a^{-1}\left[\bar{\gamma}^{a b} \delta \Gamma_{\nu \lambda}^{\varphi} \bar{X}_{, a}^{\nu} \bar{X}_{, b}^{\lambda}+\left(\delta^{(3)} \Delta\right) \bar{\varphi}+\delta \gamma^{a b} \bar{\Gamma}_{\nu \lambda}^{\varphi} \bar{X}_{, a}^{\nu} \bar{X}_{, b}^{\lambda}\right]=0$.

The non-vanishing changes of the metric induced on the brane are

$$
\begin{array}{ll}
\delta \gamma_{v \theta}=-a \frac{r_{0}}{r} \sin ^{2} \theta \bar{\varphi}_{, \theta}, & \delta \gamma_{r \theta}=a \sin ^{2} \theta\left(-1+r^{2} \psi_{, r}\right) \bar{\varphi}_{, \theta}, \\
\delta \gamma_{\theta \theta}=2 a r^{2} \sin ^{2} \theta \psi_{, \theta} \bar{\varphi}_{, \theta} . &
\end{array}
$$

The calculations of variations which enter (3.8) are straightforward but rather long. The calculations give the following equation for $\psi$ :

$$
\text { (3) } \bar{\Delta} \psi+\frac{2}{r^{2}} \cot \theta \psi_{, \theta}+2 \frac{B}{r} \psi_{, r}=\frac{1}{r^{3}} \text {. }
$$

If $\psi$ is a function of $r$ only, this equation takes the form

$$
\partial_{r}\left(r B \psi^{\prime}\right)+2 B \psi^{\prime}=\frac{1}{r^{2}} .
$$

Its solution which is regular at the horizon and at infinity is

$$
\psi=-\frac{1}{r} \text {. }
$$

We demonstrate now that this is the only regular solution of (3.10).

\subsection{Uniqueness of a regular solution}

A solution of (3.10) is a sum of (3.12), and a solution of the homogeneous equation

$$
{ }^{(3)} \bar{\Delta} \tilde{\psi}+\frac{2}{r^{2}} \cot \theta \tilde{\psi}_{, \theta}+2 \frac{B}{r} \tilde{\psi}_{, r}=0 \text {. }
$$

We show that it does not have nontrivial solutions which are regular both at the horizon and infinity.

Note that this equation allows a separation of variables. By writing a solution in the form $\tilde{\psi}(r, \theta)=\eta(r) \chi(\theta)$ and substituting it into the equation (3.13), one obtains

$$
\begin{aligned}
& \frac{\mathrm{d}}{\mathrm{d} r}\left(r^{3} B \frac{\mathrm{d} \eta}{\mathrm{d} r}\right)=\Lambda r \eta, \\
& \frac{\mathrm{d}}{\mathrm{d} \theta}\left(\frac{\Psi^{3}}{\sin \theta} \frac{\mathrm{d} \chi}{\mathrm{d} \theta}\right)=-\Lambda \sin \theta \Psi \chi .
\end{aligned}
$$

Here $\Lambda$ is a separation constant and

$$
\Psi=\sqrt{\sin ^{2} \theta-\sin ^{2} \alpha} .
$$

At the boundary points of the interval $(\alpha, \pi-\alpha)$ the function $\Psi$ vanishes, and the angular equation (3.15) has singular points. For a regular solution, $\chi$ remains finite at these boundary points. The corresponding boundary value problem determines the spectrum $\Lambda$. By multiplying the angular equation (3.15) by $\chi$, integrating it over the interval $(\alpha, \pi-\alpha)$ and making the integration by parts, one obtains

$$
\int_{\alpha}^{\pi-\alpha} \frac{\mathrm{d} \theta}{\sin \theta} \Psi^{3}\left(\frac{\mathrm{d} \chi}{\mathrm{d} \theta}\right)^{2}=\Lambda \int_{\alpha}^{\pi-\alpha} \mathrm{d} \theta \sin \theta \Psi \chi^{2} .
$$

This relation implies that the separation constant $\Lambda$ is non-negative. 
Consider now solutions of the radial equation (3.14). It is easy to see that these solutions at large $r$ have the following asymptotic: $\eta(r) \sim r^{\alpha}$, where $\alpha(\alpha+2)=\Lambda$. Since $\Lambda \geqslant 0$ one has either $\alpha \geqslant 0$ or $\alpha \leqslant-2$. In the first case the only solution which remains finite at infinity is $\tilde{\psi}=$ const. This solution is trivial and can be absorbed by a change of position of the unperturbed domain wall $\varphi \rightarrow \varphi+$ const. For all other solutions regular at infinity one has $\alpha \leqslant-2$. The equality takes place only for $\Lambda=0$.

By multiplying the radial equation (3.14) by $\eta$, integrating it over the interval $\left(r_{0}, \infty\right)$, and making the integration by parts one obtains

$$
\Lambda \int_{r_{0}}^{\infty} \mathrm{d} r r \eta^{2}+\int_{r_{0}}^{\infty} \mathrm{d} r r^{3} B\left(\frac{\mathrm{d} \eta}{\mathrm{d} r}\right)^{2}=\left.r^{3} B \eta \frac{\mathrm{d} \eta}{\mathrm{d} r}\right|_{r_{0}} ^{\infty} .
$$

The expression on the right-hand side vanishes both at the horizon (where $B=0$ ) and at the infinity. Both integrals on the left-hand side are non-negative. The equality is possible only if $\eta=0$. This proves that the homogeneous equation (3.13) does not have nontrivial solutions.

This result allows also the following interpretation. In the unperturbed static space the induced metric on the domain wall is the metric of a static $(2+1)$-dimensional black hole. The uniqueness theorem we proved can be interpreted as a statement that this $(2+1)$-dimensional black hole does not have 'hairs' of a scalar field $\tilde{\psi}$.

Thus (3.12) is a unique regular solution. The result of our computations for the vector $\delta X^{\mu}$ which describes deformation of the position of the domain wall can be represented in a more geometrical form. Let $\xi_{(\phi)}=\partial_{\varphi}$ be the Killing vector of the Kerr metric related to rotation. Then, our solution simply is

$$
\delta X^{\mu}=-\frac{a}{r} \xi_{(\phi)}^{\mu} .
$$

\subsection{Angular momentum flux}

We show now that the rotation induces the angular momentum flux which is transferred from the black hole to the domain wall. To calculate this flux we use the relation (2.8) which in the case of a domain wall is

$$
\dot{j}^{b} \equiv \frac{\mathrm{d} J^{b}}{\mathrm{~d} v}=\int_{r=\text { const }} \sqrt{-g} T_{\varphi}^{r} \mathrm{~d}^{2} \Omega .
$$

For the regular solution (3.12)

$$
\sqrt{-g} T_{\varphi}^{r}=\sigma r_{0} a \sin \theta \sqrt{\sin ^{2} \theta-\sin ^{2} \alpha} \delta(\varphi-\bar{\varphi}(\theta)) .
$$

The rate of change of the angular momentum of the black hole $J=a M$ is

$$
\dot{J}=-\pi \sigma a r_{0} \cos ^{2} \alpha=-2 \pi G_{4} \sigma \cos ^{2} \alpha J .
$$

The angular momentum flux vanishes when the domain wall is in the equatorial plane of the rotating black hole ${ }^{5}$. This is the final axisymmetric stationary equilibrium configuration of the rotating black hole in the presence of the domain wall. The relaxation time when the black hole reaches this final state is $T \sim\left(\pi G_{4} \sigma \cos ^{2} \alpha\right)^{-1}$.

In appendix we present similar results for a rotating Kerr black hole attached to a cosmic string. Since Nambu-Goto equations for a test stationary string allow an exact solution for an arbitrary value of the rotation parameter, it is possible to obtain an exact expression for the angular momentum flux beyond the slow rotation approximation. The flux of the angular momentum vanishes only if the string is directed along the axis of rotation of the black hole so that the final state is axisymmetric.

5 It is easy to check that for an arbitrary value of $a$ the equatorial plane of the Kerr black hole is a geodesic surface and, hence, it obeys Nambu-Goto equations. 


\section{Interaction of higher-dimensional rotating black holes with branes}

\subsection{Myers-Perry metric}

Now we consider a general case. We assume that an $N$-dimensional rotating black hole is attached to an $n$-dimensional brane. If the black hole size is much smaller that the size of extra dimensions, and the tension of the brane is small, the gravitational field of the black hole is described by the Myers-Perry (MP) metric [6]. This metric, besides the timelike at infinity Killing vector $\xi_{(t)}^{\mu}$, has $[N / 2]$ (the integer part of $N / 2$ ) mutually commutative and mutually orthogonal spacelike Killing vectors $\xi_{(i)}^{\mu}$ singled out by the property that they have closed integral lines. The Killing vectors $\xi_{(i)}$ are elements of the Cartan sub-algebra of the group of rotations $S O(n)$. The MP metric is characterized by the gravitational radius $r_{+}$and by $[N / 2]$ rotation parameters $a_{i}$. Such a black hole has angular velocities $\Omega_{i}=a_{i} /\left(r_{+}^{2}+a_{i}^{2}\right)$. The vector

$$
\eta=\xi_{(t)}+\sum_{i} \Omega_{i} \xi_{(i)}
$$

at the horizon becomes the null generator of the horizon. (The summation over $i$ is performed from $i=1$ to $i=[N / 2]$.)

The metric takes a slightly different form for even and odd $N$. For even $N$ the MP metric in the ingoing coordinates is

$$
\begin{aligned}
\mathrm{d} s^{2}=-\mathrm{d} v^{2}+ & 2 \mathrm{~d} v \mathrm{~d} r+\sum_{i=1}^{N / 2}\left[\left(r^{2}+a_{i}^{2}\right)\left(\mathrm{d} \mu_{i}^{2}+\mu_{i}^{2} \mathrm{~d} \varphi_{i}^{2}\right)\right. \\
& \left.-2 a_{i} \mu_{i}^{2} \mathrm{~d} r \mathrm{~d} \varphi_{i}+\frac{m r^{2}}{\Pi F}\left(\mathrm{~d} v-a_{i} \mu_{i}^{2} \mathrm{~d} \varphi_{i}\right)^{2}\right]
\end{aligned}
$$

where

$$
\Pi=\prod_{i=1}^{[N / 2]}\left(r^{2}+a_{i}^{2}\right), \quad F=1-\sum_{i=1}^{[N / 2]} \frac{a_{i}^{2} \mu_{i}^{2}}{r^{2}+a_{i}^{2}} .
$$

The $\mu_{i}$ are coordinates on a unit $[N / 2]$-dimensional sphere

$$
\sum_{i=1}^{[N / 2]} \mu_{i}^{2}=1
$$

The $\varphi_{i} \mathrm{~s}$ are angles specifying the direction in the bi-planes of rotation. The Killing vectors of MP metric are $\xi_{(i)}=\partial_{\varphi_{i}}$ The parameter $m=r_{0}^{N-2}$ is related to the mass $M$ of the black hole, see (2.5). The angular momenta $J_{i}$ of the black hole are defined as

$$
J_{i}=\frac{\mathcal{A}_{N-1}}{8 \pi G^{N+1}} m a_{i}=\frac{2}{N-1} M a_{i} .
$$

The position of the event horizon $r_{+}$is defined by the equation

$$
\Pi-m r^{2}=0 \text {. }
$$

The solution for odd $N$ is ${ }^{6}$

$$
\begin{aligned}
& \mathrm{d} s^{2}=-\mathrm{d} v^{2}+2 \mathrm{~d} v \mathrm{~d} r+r^{2} \mathrm{~d} \mu^{2}+\sum_{i=1}^{[N / 2]}\left[\left(r^{2}+a_{i}^{2}\right)\left(\mathrm{d} \mu_{i}^{2}+\mu_{i}^{2} \mathrm{~d} \varphi_{i}^{2}\right)\right. \\
& \left.-2 a_{i} \mu_{i}^{2} \mathrm{~d} r \mathrm{~d} \varphi_{i}+\frac{m r^{2}}{\Pi F}\left(\mathrm{~d} v-a_{i} \mu_{i}^{2} \mathrm{~d} \varphi_{i}\right)^{2}\right],
\end{aligned}
$$

6 It should be noted that the sign of $a_{i}$ in both of the metrics (4.2) and (4.7) is opposite to the sign adopted in the paper [6]. For our choice of signs the metric (4.7) for $N=3$ coincides with Kerr metric (A.1). 
where $\Pi$ and $F$ are defined by (4.3) and $\mu$ and $\mu_{i}$ obey the following relation:

$$
\mu^{2}+\sum_{i=1}^{[N / 2]} \mu_{i}^{2}=1
$$

The mass and angular momenta are defined as for the even $N$ case, while a location of the event horizon is determined by the equation

$$
\Pi-m r=0 .
$$

In the absence of rotation both metrics (4.2) and (4.7) reduce to the Tangherlini metric (2.4).

\subsection{Slow rotation limit}

Our aim is to investigate interaction of branes with slowly rotating black holes. For slow rotation, $a_{i} / r_{0} \ll 1$, both metrics (4.2) and (4.7) can be written in a form similar to (3.1)

$$
\mathrm{d} s^{2}=\mathrm{d} \bar{s}^{2}-2\left[\mathrm{~d} r+\left(\frac{r_{0}}{r}\right)^{N-2} \mathrm{~d} v\right] \sum_{i=1}^{[N / 2]} a_{i} \mu_{i}^{2} \mathrm{~d} \varphi_{i} .
$$

Here $\mathrm{d} \bar{s}^{2}$ is the unperturbed metric (2.4). The metric on the unit sphere $\mathrm{d} \Omega_{N-1}^{2}$ in (2.4) has the form

$$
\begin{aligned}
& \mathrm{d} \Omega_{N-1}^{2}=\sum_{i=1}^{[N / 2]}\left(\mathrm{d} \mu_{i}^{2}+\mu_{i}^{2} \mathrm{~d} \varphi_{i}^{2}\right), \quad \text { for } \quad N \text { even, } \\
& \mathrm{d} \Omega_{N-1}^{2}=\mathrm{d} \mu^{2}+\sum_{i=1}^{[N / 2]}\left(\mathrm{d} \mu_{i}^{2}+\mu_{i}^{2} \mathrm{~d} \varphi_{i}^{2}\right), \quad \text { for } \quad N \text { odd } .
\end{aligned}
$$

By taking into account (2.4), (4.11) and (4.12), one can represent (4.10) as

$$
\begin{aligned}
& \mathrm{d} s^{2}=\mathrm{d} \bar{s}^{2}-\frac{2}{r^{2}}\left[\mathrm{~d} r+\left(\frac{r_{0}}{r}\right)^{N-2} \mathrm{~d} v\right] \varrho_{\mu} \mathrm{d} x^{\mu}, \\
& \varrho_{\mu}=\bar{g}_{\mu \nu} \varrho^{\nu}, \quad \varrho^{\mu}=\sum_{i} a_{i} \xi_{(i)}^{\mu} .
\end{aligned}
$$

In the linear approximation $r_{+} \approx r_{0}, \Omega_{i} \approx a_{i} / r_{0}^{2}$, and instead of (4.1) one has

$$
\eta=\xi_{(t)}+r_{0}^{-2} \varrho \text {. }
$$

Therefore, in the linear approximation $\varrho$ is related to the vector $\eta$ which at the horizon becomes the null generator of the horizon surface.

\subsection{Solution to the linearized Nambu-Goto equations}

Let us consider a brane interacting with a higher-dimensional slowly rotating black hole described by metric (4.10). As we have already pointed out the rotation results in the deformation of the brane worldsheet described by the vector field $\delta X^{\mu}$. This deformation is the solution to linearized Nambu-Goto equations (2.10). We demonstrate now that these equations allow a solution of the form $\delta X^{\mu}=\psi \varrho^{\mu}$, where $\psi=\psi(r)$ and $\varrho^{\mu}$ is the Killing vector field (4.14). It is this form of solution which we found for the domain wall in case of Kerr black hole. 
By using (4.10) one can show that in coordinates where $\xi_{(i)}^{\mu}=\delta_{\varphi_{i}}^{\mu}$, the following relations are valid:

$$
\begin{aligned}
& { }^{(n+1)} \bar{\Delta} \delta X^{\mu}=\frac{1}{r^{n-1}} \partial_{r}\left(r^{n-1} B \psi^{\prime}\right) \varrho^{\mu} \\
& \left(\delta^{(n+1)} \Delta\right) \bar{X}^{\mu}=\frac{1}{r^{n-1}} \partial_{r}\left(r^{n-1}\left(\frac{1}{r^{2}}-B \psi^{\prime}\right)\right)\left(\varrho^{\|}\right)^{\mu} \\
& 2 \bar{\gamma}^{a b} \bar{\Gamma}_{\nu \lambda}^{\mu} \bar{X}_{, a}^{\lambda} \delta X_{, b}^{v}=\frac{2 B}{r} \psi^{\prime} \varrho^{\mu} \\
& \delta \gamma^{a b} \bar{\Gamma}_{\nu \lambda}^{\mu} \bar{X}_{, a}^{\lambda} \bar{X}_{, b}^{v}=\frac{2}{r}\left(\frac{1}{r^{2}}-B \psi^{\prime}\right)\left(\varrho^{\|}\right)^{\mu}, \\
& \bar{\gamma}^{a b} \delta \Gamma_{\nu \lambda}^{\mu} \bar{X}_{, a}^{\lambda} \bar{X}_{, b}^{v}=-\frac{n-1}{r^{3}} \varrho^{\mu} .
\end{aligned}
$$

Here $\varrho^{\|}$is the projection of the vector $\varrho$ on the plane tangent to the brane. With these relations linearized, Nambu-Goto equations (2.10) take the simple form

$$
\left[\frac{1}{r^{n-1}} \partial_{r}\left(r^{n-1} B \psi^{\prime}\right)+\frac{2 B}{r} \psi^{\prime}-\frac{n-1}{r^{3}}\right]\left(\varrho^{\perp}\right)^{\mu}=0,
$$

where $\varrho^{\perp}=\varrho-\varrho^{\|}$is the projection of $\varrho$ in the hyperplane orthogonal to the brane. As follows from (4.16), if $\varrho^{\perp}=0$ the Nambu-Goto equations are satisfied automatically. In this case a deformation vector $\delta X^{\mu}$ is tangent to the brane worldsheet. Such deformations can be gauged away. If $\varrho^{\perp} \neq 0$ the square bracket in (4.16) must vanish. This condition yields the equation for the function $\psi$, which has a single solution regular both at the horizon $r=r_{0}$ and at infinity

$$
\psi(r)=-\int_{r}^{\infty} \frac{\mathrm{d} r}{r^{2} B}\left[1-\left(\frac{r_{0}}{r}\right)^{n-1}\right] .
$$

For a domain wall interacting with the Kerr black hole $(n=2)$ this expression reduces to the result obtained earlier, $\psi=-1 / r$. In the case of cosmic strings, $n=1$, the only regular solution is $\psi=0$ for any dimension $N$ of the bulk black hole.

A general solution of the linearized Nambu-Goto equations (2.10) is a sum of the obtained solution $\psi(r) \varrho^{\mu}$ and a solution of the homogeneous equations. In the case of the domain wall we proved that there is no nontrivial homogeneous solutions which are regular both at the event horizon and infinity. One can expect that in the higher-dimensional case the situation is similar. One can check that a trivial homogeneous solution is $\sum_{i}^{[N / 2]} c_{i} \xi_{(i)}^{\mu}\left(c_{i}\right.$ are constants). It can be eliminated by appropriate shifts of coordinates $\varphi_{i}$.

\subsection{Final stationary state of a rotating black hole}

We can now describe a final stationary state which is reached by a black hole as a result of the interaction with the brane. To this aim we investigate loss of the angular momentum of the black hole. Since for the static metric $\mathrm{d}^{2} \dot{J}_{i}$ vanishes, it is sufficient to calculate the variation of (2.9) induced by the metric perturbations. We have

$$
\dot{J}_{i}=\sigma \int_{r=\text { const }} \mathrm{d}^{n-1} \omega \delta\left(\sqrt{-\gamma} \gamma^{a b} X_{, a}^{\mu} n_{\mu} X_{, b}^{\nu} \xi_{(i) \nu}\right)
$$

It is easy to check that in the linear in $a_{i}$ approximation the following relations for the variations induced by the perturbed metric (4.13) are valid: $\delta(\sqrt{-\gamma})=0$,

$$
\xi_{(i) a} \delta \gamma^{r a}=\frac{1}{r^{2}}\left(\xi_{(i)}^{\|}, \varrho^{\|}\right)-B\left(\xi_{(i)}^{\|}, \delta X_{, r}^{\|}\right),
$$




$$
\begin{aligned}
& \gamma^{r a} \delta \xi_{(i) a}=-\frac{a_{i}}{r^{2}}\left(\xi_{(i)}\right)^{2}, \\
& \gamma^{r a} \xi_{(i) \lambda} \delta X_{, a}^{\lambda}=B\left(\xi_{(i)}, \delta X_{, r}\right) .
\end{aligned}
$$

As earlier we denote by $p^{\|}$a projection of the vector $p$ on the brane. $(p, q)$ is a scalar product of vectors $p$ and $q$ in the unperturbed metric, $(p, q)=\bar{g}_{\mu \nu} p^{\mu} q^{\nu}$.

The flux of the angular momenta from the black hole to the brane changes the angular momenta of the black hole (4.5). In the linear approximation the equations for the change of the angular momenta of the black hole can be written as follows:

$$
\dot{\mathbf{J}}=-\mathbf{K J} \text {. }
$$

We use bold-faced quantities for vectors and tensors in the space of rotation parameters, so $\mathbf{J}$ and $\mathbf{K}$ have components $J_{i}$, and $K_{i j}$. Relations (4.5), (4.17)-(4.21) enable one to get $\mathbf{K}$ in the form

$$
\begin{aligned}
& K_{i j}=(N-1) \sigma r_{0}^{n-1} k_{i j} /(2 M), \\
& k_{i j}=\int_{S^{n-1}} \mathrm{~d} \omega^{n-1} \frac{1}{r^{2}}\left(\xi_{(i)}^{\perp}, \xi_{(j)}^{\perp}\right) .
\end{aligned}
$$

We denote by $p^{\perp}$ a projection of a vector $p$ orthogonal to the brane. In an agreement with the conservation law, the angular momentum flux does not depend on the radius $r$ of the surface where it is calculated.

Note that in the linear in $a_{i}$ approximation $\dot{M}=\dot{r}_{0}=0$ and $M$ and $r_{0}$ in (4.23) are considered as constant parameters. The evolution equation (4.5) can also be written as $\dot{\mathbf{a}}=-\mathbf{K a}$, where $\mathbf{a}$ is a vector with components $a_{i}$. This equation shows that the black hole can be stationary, $\mathbf{a}=0$, if and only if $\mathbf{a}$ is the zero vector, $\mathbf{K a}=0$. In this case the equation $\mathbf{a}^{T} \mathbf{K a}=0$ implies that

$$
\int_{S^{n-1}} \mathrm{~d} \omega^{n-1}\left(\varrho^{\perp}, \varrho^{\perp}\right)=0
$$

and hence $\varrho^{\perp}=0$. This means that the corresponding Killing vector $\varrho$ generates transformations that preserve a position of the brane. The stationary metric of the final black hole configuration in this case is given by (4.13), where $\varrho$ is a vector tangent to the brane. Since in the linear approximation $\varrho$ is related to the null generator of the black hole horizon, $\eta=\xi_{(t)}+r_{0}^{-2} \varrho$, in the final state of the black hole $\eta$ is tangent to the brane.

This property is valid for any value of a rotation parameter. In the stationary case the surface area of the black hole horizon is constant. By using the Raychaudhuri equation one has $T_{\mu \nu} \eta^{\mu} \eta^{\nu}=0$, where $T_{\mu \nu}$ is the stress-energy tensor of the brane. Since $T_{\mu \nu}$ is proportional to the induced metric, the null generator of the horizon $\eta$ is tangent to the brane on the horizon ${ }^{7}$.

However, the result we have found for slowly rotating black holes is more than that. We have shown that vector fields $\eta$ and $\varrho$ are tangent everywhere to the brane worldsheet, not only at the horizon. Moreover, because the deformation of the brane has the form $\delta X^{\mu}=\psi(r) \varrho^{\mu}$, it follows that in the final stationary state $\delta X^{\perp \mu}=0$. Since the tangent to the brane components of $\delta X^{\mu}$ can always be gauged away, in the limit of slow rotation the final state of a stationary brane is not deformed. It is easy to see that the condition that $\varrho$ is tangent to the brane implies that the brane stress-energy tensor obeys the condition $\mathcal{L}_{\varrho} T^{\mu \nu}=0$. This is a result of the invariance of the brane under the symmetry transformations generated by $\varrho$. In the $N=3$ case this is a condition of the axial symmetry, in accordance with the Hawking theorem [9].

\footnotetext{
7 A similar condition for a static black hole was discussed in [11] in a slightly different context.
} 


\section{Evolution equations for special cases}

In a general case, the evolution in the space of the rotation parameters and a number of free rotation parameters available in the final state are determined by the properties of the matrix k. From a mathematical point of view, this is a problem of mutual orientation of a Cartan subalgebra of fields $\xi_{i}$ related to the rotation planes of the black hole, and a sphere $S^{n-1} \subset S^{N-1}$ related to the brane position. To illustrate different possibilities we consider some special cases.

We consider a unit sphere $S^{N-1}$ embedded in the Euclidean space $R^{N}$ and let $X^{A}, A=$ $1, \ldots, N$, be the Cartesian coordinates in $R^{N}$. A sphere $S^{n-1}$ representing the position of the brane can be obtained as an intersection of an $n$-dimensional hyperplane $P_{n}$ with $S^{N-1}$. We choose the coordinates $X^{A}$ so that the plane $P_{n}$ is described by the equations $X^{n+1}=\cdots=X^{N}=0$. We denote by $\vec{e}_{A}=\partial /\left(\partial X^{A}\right)$ the basis vectors in $R^{N}$. The first $n$ vectors span $P_{n}$, while the last $N-n$ vectors of this basis are orthogonal to $P_{n}$. Any vector $\vec{v}$ in $R^{N}$ is a sum of its projections parallel $\left(\vec{v}^{\|}\right)$and orthogonal $\left(\vec{v}^{\perp}\right)$ to the hyperplane $P_{n}$.

The Cartan sub-algebra for $S O(N)$ can be specified by choosing [N/2] mutually orthogonal bi-planes. Let $\vec{p}_{i}$ and $\vec{q}_{i}$ be two orthonormal vectors which span the $i$ th bi-plane of rotation. We denote by

$$
(\vec{v} \cdot \vec{u})=\sum_{A=1}^{N} v_{A} u_{A}
$$

a scalar product of two vectors $\vec{v}$ and $\vec{u}$ in $R^{N}$. By definition

$$
\left(\vec{p}_{i} \cdot \vec{p}_{j}\right)=\left(\vec{q}_{i} \cdot \vec{q}_{j}\right)=\delta_{i j}, \quad\left(\vec{p}_{i} \cdot \vec{q}_{j}\right)=0 .
$$

A Killing vector field $\vec{\xi}_{(i)}$ generating a rotation in a given bi-plane can be written as

$$
\vec{\xi}_{(i)}=\left(\vec{p}_{i} \cdot \vec{X}\right) \vec{q}_{i}-\left(\vec{q}_{i} \cdot \vec{X}\right) \vec{p}_{i}
$$

Let us denote

$$
\vec{\zeta}_{(i)}=\left.\vec{\xi}_{(i)}^{\perp}\right|_{S^{n-1}}
$$

then we have

$$
\vec{\zeta}_{(i)}=\left(\vec{p}_{i}^{\|} \cdot \vec{X}\right) \vec{q}_{i}^{\perp}-\left(\vec{q}_{i}^{\|} \cdot \vec{X}\right) \vec{p}_{i}^{\perp} .
$$

Since $\vec{X}$ in this relation belongs to $S^{n-1}$, one has

$$
(\vec{X} \cdot \vec{X})=\sum_{A=1}^{n} X^{A} X^{A}=1 .
$$

The scalar product which enters the matrix $k_{i j}$, see (4.24), is

$$
\frac{1}{r^{2}}\left(\xi_{(i)}^{\perp}, \xi_{(j)}^{\perp}\right)=\left(\vec{\zeta}_{(i)} \cdot \vec{\zeta}_{(j)}\right)
$$

After integrating over $S^{n-1}$ one finds

$$
\begin{aligned}
& k_{i j}=\int_{S^{n-1}} \mathrm{~d} \omega^{n-1}\left(\vec{\zeta}_{(i)} \cdot \vec{\zeta}_{(j)}\right)=Z_{n}\left[\left(\vec{q}_{i}^{\perp} \cdot \vec{q}_{j}^{\perp}\right)\left(\vec{p}_{i}^{\|} \cdot \vec{p}_{j}^{\|}\right)\right. \\
& \left.+\left(\vec{p}_{i}^{\perp} \cdot \vec{p}_{j}^{\perp}\right)\left(\vec{q}_{i}^{\|} \cdot \vec{q}_{j}^{\|}\right)-\left(\vec{q}_{i}^{\perp} \cdot \vec{p}_{j}^{\perp}\right)\left(\vec{p}_{i}^{\|} \cdot \vec{q}_{j}^{\|}\right)-\left(\vec{p}_{i}^{\perp} \cdot \vec{q}_{j}^{\perp}\right)\left(\vec{q}_{i}^{\|} \cdot \vec{p}_{j}^{\|}\right)\right] .
\end{aligned}
$$

Here we used the fact that

$$
\int_{S^{n-1}} \mathrm{~d} \omega^{n-1} X^{A} X^{B}=\delta^{A B} Z_{n}, \quad Z_{n}=\frac{\pi^{n / 2}}{\Gamma\left(\frac{n+2}{2}\right)} .
$$

Formula (5.7) enables one to infer properties of the matrix $k_{i j}$. Let us discuss some simple cases. 
(1) Suppose the $i$ th bi-plane of rotation has the orientation for which $\vec{p}_{i}^{\|}=\vec{q}_{i}^{\|}=0$, then the components of $\mathbf{k}$ with a chosen index $i$ vanish. The rotation in the given $i$ th bi-plane transforms the brane into itself. There are no fluxes of the $i$ th component of the angular momentum, $\dot{J}_{i}=0$. An example of this case is a cosmic string directed along the rotation axis of the Kerr black hole.

(2) Suppose the $i$ th bi-plane has the orientation for which $\vec{p}_{i}^{\perp}=\vec{q}_{i}^{\perp}=0$. Then again, there is no flux of $J_{i}, \dot{J}_{i}=0$, and the rotation in the $i$ th bi-plane transforms the brane into itself. An example of this situation is a domain wall lying in the equatorial plane of the Kerr black hole.

(3) Suppose that $\vec{q}_{i}^{\|}=0$ but $\vec{p}_{i}^{\|} \neq 0$. Then $\left(\vec{q}_{i}^{\perp} \cdot \vec{q}_{j}^{\perp}\right)=\left(\vec{q}_{i} \cdot \vec{q}_{j}\right)=\delta_{i j},\left(\vec{q}_{i}^{\perp} \cdot \vec{p}_{j}^{\perp}\right)=\left(\vec{q}_{i} \cdot \vec{p}_{j}\right)=0$ and

$$
k_{i j}=Z_{n} \delta_{i j} \sin ^{2} \alpha_{i} \quad \text { for any } j,
$$

where $\sin ^{2} \alpha_{i} \equiv\left(\vec{p}_{i}^{\|} \cdot \vec{p}_{i}^{\|}\right)$. The parameter $\alpha_{i}$ is related to the angle $\pi / 2-\alpha_{i}$ between the brane and the $i$ th bi-plane. This configuration is realized when a cosmic string is tilted to the rotation axis of the Kerr black hole. In this case $\alpha_{i}$ is the angle between the string and the axis. The flux of the angular momentum is proportional to $\sin ^{2} \alpha_{i}$, in agreement with (A.12).

(4) Suppose that $\vec{q}_{i}^{\perp}=0$ but $\vec{p}_{i}^{\perp} \neq 0$. Then $\left(\vec{q}_{i}^{\|} \cdot \vec{q}_{j}^{\|}\right)=\delta_{i j},\left(\vec{q}_{i}^{\|} \cdot \vec{p}_{j}^{\|}\right)=0$ and

$$
k_{i j}=\delta_{i j} \cos ^{2} \alpha_{i} Z_{n} \quad \text { for any } j .
$$

For the domain wall tilted at the angle $\alpha_{i}$ to rotation axis of the Kerr black hole one gets the momentum flux proportional to $\cos ^{2} \alpha_{i}$, in agreement with (3.22).

The above examples show that the matrix $k_{i j}$ can have eigenvectors with positive eigenvalues. These eigenvectors define the directions in the space of parameters $a_{i}$ for which the evolution is damping. The damping is caused by the 'friction' which is a result of the interaction between the black hole and the brane.

\section{Discussion}

Let us summarize the results obtained in this paper. We considered interaction of branes with rotating higher-dimensional black holes. Such systems include several physically interesting examples, such as cosmic strings and thin domain walls interacting with a Kerr black hole, as well as rotating black holes in a spacetime with large extra dimensions. If a black hole does not rotate, in the zero order in the string tension (test string approximation) a static brane located in the black hole's 'equatorial plane' is always a solution of the Nambu-Goto equations. In the presence of rotation, a stationary brane attached to a black hole is deformed. In a general case, this deformation $\delta X^{\mu}=\psi(r) \varrho^{\mu}$ is determined by the Killing vector $\varrho^{\mu},(4.14)$, which describes the change of the black hole metric under slow rotation, see (4.13). For a Kerr black hole, we demonstrated that these solutions obeying the regularity conditions at the horizon and infinity are unique. One can make a conjecture that this property is valid for an arbitrary number of space and brane dimensions.

We demonstrated that in the general case there exists an angular momentum transfer from a black hole to the attached brane until $\varrho$ becomes tangent to the brane. The brane stressenergy tensor in this state obeys condition $\mathcal{L}_{\varrho} T^{\mu \nu}=0$. One may expect that there should exist a generalization of this result for a rotating black hole surrounded by a stationary distribution of matter or field ${ }^{8}$, so that a higher-dimensional analogue of the Hawking theorem [9] is valid.

8 One of the possible examples is a five-dimensional rotating black hole in a homogeneous magnetic field; see [12]. 
The flux of the angular momentum is determined by the tension of the brane $\sigma$ and by the black hole radius $r_{0}$

$$
\dot{J} \sim-G \sigma r_{0}^{1-p} J,
$$

where $p$ is the number of brane co-dimensions dimensions. The characteristic time of the relaxation process during which the black hole reaches its final state can be estimated by using (6.1)

$$
T \sim r_{0}^{p-1} /\left(G_{N+1} \sigma\right) \sim T_{*}\left(r_{0} / L_{*}\right)^{p-1}\left(\sigma_{*} / \sigma\right) .
$$

Here $p=N-n$ is the number of extra dimensions, $\sigma_{*}=M_{*} / L_{*}^{n}$ and quantities $M_{*}, L_{*}$ are, respectively, the fundamental mass and the length of the theory.

It should be noted that the black hole can also lose its bulk components of the rotation by emitting Hawking quanta in the bulk. The characteristic time of this process is $T_{H} \sim T_{*}\left(r_{0} / L_{*}\right)^{N}$. For black holes which can be treated classically $r_{0} \gg L_{*}, T_{H} \gg T$. Thus the friction effect induced by the brane is the dominant one.

We would like to conclude the paper by the following remark. We focused our attention on the higher-dimensional spacetimes with vanishing bulk cosmological constant (ADD model [1]). A similar problem concerning general properties of higher-dimensional rotating black holes with the horizon radius $r_{0}$ can be addressed in the Randall-Sundrum (RS) models [2] provided the bulk cosmological constant is much smaller than $r_{0}^{-2}$. A characteristic property of such models is the existence of $Z_{2}$ symmetry. Under the $Z_{2}$ transformation the brane remains unchanged, while the components of any vector orthogonal to the brane change their sign. Thus $Z_{2}$ symmetry implies $Q^{\perp}=0$. Hence a stationary black hole attached to the brane in the RS-model can rotate only within the brane.

The relaxation process related to the presence of $\varrho^{\perp}$ which is typical for the ADD-model is absent in the RS-model. This is an additional signature which in principle may allow one to distinguish between these models in observations.

\section{Acknowledgments}

VF and DF kindly acknowledge support from the NATO Collaborative Linkage grant 979723. The work of VF and DF is also partially supported by the Killam Trust and the Natural Sciences and Engineering Research Council of Canada.

\section{Appendix. The interaction of a Kerr black hole with a cosmic string}

In this appendix we consider the interaction of a Kerr black hole with a cosmic string. This case is of special interest since the stationary test string equations can be solved exactly for an arbitrary value of the rotation parameter $a$. The Kerr metric in the Boyer and Lindquist coordinates is

$\mathrm{d} s^{2}=-\left(1-\frac{2 M r}{\Sigma}\right) \mathrm{d} t^{2}-\frac{4 M r a \sin ^{2} \theta}{\Sigma} \mathrm{d} t \mathrm{~d} \phi+\frac{\Sigma}{\Delta} \mathrm{d} r^{2}+\Sigma \mathrm{d} \theta^{2}+\frac{A \sin ^{2} \theta}{\Sigma} \mathrm{d} \phi^{2}$,

where

$\Sigma \equiv r^{2}+a^{2} \cos ^{2} \theta, \quad \Delta \equiv r^{2}-2 M r+a^{2}, \quad A=\left(r^{2}+a^{2}\right)^{2}-a^{2} \Delta \sin ^{2} \theta$.

The event horizon is located at $r=r_{+}=M+\sqrt{M^{2}-a^{2}}$.

By making the coordinate transformation

$$
\mathrm{d} v=\mathrm{d} t+\left(r^{2}+a^{2}\right) \frac{\mathrm{d} r}{\Delta}, \quad \mathrm{d} \varphi=\mathrm{d} \phi+a \frac{\mathrm{d} r}{\Delta},
$$


we obtain this metric in the Kerr ingoing coordinates

$$
\begin{gathered}
\mathrm{d} s^{2}=-\left[1-\Sigma^{-1}(2 M r)\right] \mathrm{d} v^{2}+2 \mathrm{~d} r \mathrm{~d} v+\Sigma \mathrm{d} \theta^{2}+\Sigma^{-1}\left[\left(r^{2}+a^{2}\right)^{2}-\Delta a^{2} \sin ^{2} \theta\right] \sin ^{2} \theta \mathrm{d} \varphi^{2} \\
-2 a \sin ^{2} \theta \mathrm{d} \varphi \mathrm{d} r-4 a \Sigma^{-1} M r \sin ^{2} \theta \mathrm{d} \varphi \mathrm{d} v .
\end{gathered}
$$

These coordinates are regular on the future event horizon. One also has $\sqrt{-g}=\Sigma \sin \theta$. The Kerr metric possesses two Killing vectors $\xi_{(t)}^{\mu}=\delta_{v}^{\mu}$ and $\xi_{(\phi)}^{\mu}=\delta_{\varphi}^{\mu}$.

In what follows, we shall consider stationary fluxes of energy and angular momentum of some distribution of matter through a surface $r=$ const

$$
\Delta E=\int T_{\mu}^{v} \xi_{(t)}^{\mu} \mathrm{d} \sigma_{v}, \quad \Delta J=-\int T_{\mu}^{v} \xi_{(\varphi)}^{\mu} \mathrm{d} \sigma_{v} .
$$

Here $T_{\mu \nu}$ is the stress-energy tensor of the matter and

$$
\mathrm{d} \sigma_{\mu}=r_{, \mu} \sqrt{-g} \mathrm{~d} v \mathrm{~d} \theta \mathrm{d} \varphi .
$$

For a stationary configuration the (constant) rate of energy and angular momentum fluxes from the black hole through the $r=$ const surface are

$$
\dot{E} \equiv \frac{\mathrm{d} E}{\mathrm{~d} v}=\int \mathrm{d} \theta \mathrm{d} \varphi \sqrt{-g} T_{v}^{r}, \quad \dot{J} \equiv \frac{\mathrm{d} J}{\mathrm{~d} v}=-\int \mathrm{d} \theta \mathrm{d} \varphi \sqrt{-g} T_{\varphi}^{r} .
$$

If a part of initially infinite string is captured by a black hole, its worldsheet in the black hole exterior consists of two segments. Let us consider a case when a cosmic string is stationary. In [13] it was proved that any segment of this string in the Kerr spacetime which starts at infinity and crosses the infinite redshift surface, remaining regular there, has a worldsheet generated by the Killing vector $\xi_{(t)}^{\mu}$ and a principal null geodesic. The string segment regularly crosses the future event horizon. In the incoming Kerr coordinates its equation has the following simple form $\theta=$ const and $\varphi=$ const. Without loss of generality, we put $\varphi=0$, so that the string segment is described by the equations

$$
v=\hat{v}, \quad r=\hat{r}, \quad \theta=\alpha, \quad \varphi=0,
$$

where $\hat{r}, \hat{v}$ are coordinates on the string worldsheet. We denote by $\alpha$ an angle between the string segment and the rotation axis as measured at infinity. We consider a configuration where there is another string segment attached to the black hole with $\theta=\pi-\alpha, \varphi=\pi$. Because of the symmetry $\theta \rightarrow \pi-\theta$ and $\phi \rightarrow \pi+\phi$ of the Kerr metric, the strings' tension for such a symmetric configuration does not result in the accelerated motion of the black hole as a whole.

The metric induced on the string worldsheet is

$$
\gamma_{a b} \mathrm{~d} \zeta^{a} \mathrm{~d} \zeta^{b}=-B \mathrm{~d} v^{2}+2 \mathrm{~d} r \mathrm{~d} v, \quad B=1-\frac{r_{0} r}{r^{2}+a^{2} \cos ^{2} \alpha} .
$$

For a stationary configuration, the (constant) rate of angular momentum flux through the $r=$ const surface is

$$
\dot{J} \equiv \frac{\mathrm{d} J}{\mathrm{~d} v}=-\int \mathrm{d} \theta \mathrm{d} \varphi \sqrt{-g} T_{\varphi}^{r}
$$

where

$$
\sqrt{-g} T_{\varphi}^{r}=\sigma a \sin ^{2} \alpha \delta(\varphi) \delta(\theta-\alpha) .
$$

The angular momentum flux remains the same for any $r$ and is given by the following relation:

$$
\dot{J}=-2 \sigma a \sin ^{2} \alpha=-\frac{4 \sigma \sin ^{2} \alpha}{r_{0}} J .
$$

The coefficient 2 appears since there are two string segments. $\dot{J}$ vanishes when the direction of the string segments coincides with the symmetry axis, $\alpha=0$ or $\alpha=\pi$.

This result implies that the black hole loses its angular momentum whenever the string does not coincide with the axis of the rotation. In other words, in the final equilibrium configuration the black hole is either non-rotating or its angular momentum is directed along the string. The characteristic time of this process estimated from (A.12) is $T \sim r_{0} /\left(4 \sigma \sin ^{2} \alpha\right)$. 


\section{References}

[1] Arkani-Hamed N, Dimopoulos S and Dvali G 1998 Phys. Lett. B 429263

Antoniadis I, Arkani-Hamed N, Dimopoulos S and Dvali G 1998 Phys. Lett. B 436257

[2] Randall L and Sundrum R 1999 Phys. Rev. Lett. 833370

Randall L and Sundrum R 1999 Phys. Rev. Lett. 834690

[3] Eardley D and Giddings S B 2002 Phys. Rev. D 66044011

[4] Landsberg G 2003 Preprint hep-ex/0310034

[5] Kanti P 2004 Preprint hep-ph/0402168

[6] Myers R C and Perry M J 1986 Ann. Phys., NY 172304

[7] Emparan R and Reall H S 2002 Phys. Rev. Lett. 88101101

[8] Emparan R and Myers R C 2003 J. High Energy Phys. JHEP09(2003)025

[9] Hawking S W 1972 Commun. Math. Phys. 25152

[10] Tangherlini F R 1963 Nuovo Cimento 77636

[11] Emparan R, Horowitz G T and Myers R C 2000 Phys. Rev. Lett. 85499

[12] Aliev A N and Frolov V P 2004 Preprint hep-th/0401095

[13] Frolov V, Hendy S and Larsen A L 1996 Phys. Rev. D 545093 